\title{
Echocardiographic-based treatment of functional tricuspid regurgitation
}

\author{
Antonio M. Calafiore, MD, ${ }^{\mathrm{a}}$ Angela L. Iacò, MD, ${ }^{\mathrm{a}}$ Antonella Romeo, MD, ${ }^{\mathrm{b}}$ Salvatore Scandura, MD, ${ }^{\mathrm{c}}$ \\ Rocco Meduri, MD, ${ }^{\mathrm{b}}$ Egidio Varone, MD, ${ }^{\mathrm{b}}$ and Michele Di Mauro, $\mathrm{MD}^{\mathrm{d}}$
}

\begin{abstract}
Objectives: Functional tricuspid regurgitation (FTR) worsens over time, and its natural history is unfavorable. An aggressive surgical strategy, using the echocardiographic systolic dimensions of the tricuspid annulus (sysTA), can be helpful to reduce the detrimental late effects of FTR.
\end{abstract}

\begin{abstract}
Methods: From March 2006 to February 2008, 298 patients, with at least FTR grade 1+, underwent mitral valve surgery. Of these 298 patients, 167 underwent tricuspid repair (treated group [T], moderate-or-greater FTR in 108 and mild in 59, with sysTA $>24 \mathrm{~mm}$ ) and 137 did not (untreated group [UT], moderate-or-greater FTR in 16 and mild in 115; 81 with sysTA $>24 \mathrm{~mm}$ and 34 with sysTA of $\leq 24 \mathrm{~mm}$ ). The 256 survivors underwent echocardiographic examination at a mean follow-up of $13 \pm 8$ months.
\end{abstract}

Results: Preoperatively, at discharge, and at the follow-up examination, the mean FTR grade was $1.11 \pm 0.32$, $0.87 \pm 0.49$, and $1.03 \pm 0.57(P=\mathrm{NS})$ in the UT group and $2.11 \pm 0.92,0.45 \pm 0.36$, and $0.48 \pm 0.32(P<.001)$ in the T group. A total of 24 patients had FTR grade 2 or greater, $16(14.5 \%)$ in the UT group and $8(5.5 \%)$ in the T group $(P=.026)$. In the UT group, 10 of 16 patients had sysTA of 25 to $28 \mathrm{~mm}$ and 6 of 10 had sysTA greater than $28 \mathrm{~mm}$. No patient with mild FTR and sysTA of $24 \mathrm{~mm}$ or less had an increased FTR grade. Globally, 12 patients $(10.9 \%)$ had an increased FTR grade in the UT group versus none in the T group $(P<.001)$. Patients with postoperative atrial fibrillation had less residual FTR if annuloplasty had been performed ( $1.6 \pm 0.7 \mathrm{vs} 0.91$ $\pm 0.63, P=.005)$.

Conclusions: An aggressive strategy for FTR correction, using the sysTA, was able to reduce the FTR grade 1 year after surgery, but mitral surgery alone could not. (J Thorac Cardiovasc Surg 2011;142:308-13)

Functional tricuspid regurgitation (FTR) is an often neglected disease that only recently has been considered worthy of attention by surgeons. Nevertheless, no clear consensus has been reached on when FTR should be corrected and how it should be corrected. In addition, the early return of FTR is an issue that has made surgeons reluctant to expand FTR repair.

The natural history of FTR is not favorable. Nath and colleagues ${ }^{1}$ found that the survival of patients with moderate or severe FTR was significantly reduced compared with the survival of patients without or with only mild FTR. Dreyfus and colleagues ${ }^{2}$ emphasized that FTR that is even less than mild, if left untreated, can worsen by 2 grades after close to 5 years postoperatively. Because of these studies, we decided to aggressively treat FTR in patients undergoing mitral

From the Department of Adult Cardiac Surgery, ${ }^{\text {a }}$ Prince Sultan Cardiac Center, Riyadh, Kingdom of Saudi Arabia; Division of Cardiac Surgery ${ }^{\mathrm{b}}$ and Division of Cardiology, ${ }^{\mathrm{c}}$ Ferrarotto Hospital, University of Catania, Catania, Italy; and Institute of Cardiovascular Disease, ${ }^{\mathrm{d}}$ University of L'Aquila, L'Aquila, Italy.

Disclosures: Authors have nothing to disclose with regard to commercial support.

Received for publication Jan 6, 2010; revisions received March 30, 2010; accepted for publication April 16, 2010; available ahead of print Dec 16, 2010.

Address for reprints: Antonio M. Calafiore, MD, Department of Adult Cardiac Surgery, Prince Sultan Cardiac Center, Riyadh, Kingdom of Saudi Arabia (E-mail: calafiore@unich.it).

0022-5223/\$36.00

Copyright (c) 2011 by The American Association for Thoracic Surgery doi:10.1016/j.jtcvs.2010.04.047 valve (MV) surgery, according to the echocardiographic systolic dimension of the tricuspid annulus (sysTA). In the present study, we report our experience to evaluate the midterm results of our surgical strategy and to standardize the principles of surgical treatment.

\section{MATERIALS AND METHODS Population}

From March 2006 to February 2008, 298 patients with at least FTR of grade 1 underwent MV surgery. Of these 298 patients, 198 underwent repair $(66.4 \%)$ and 100 underwent replacement $(33.6 \%)$ at a single center. In addition, 167 of these patients $(56.0 \%)$ underwent tricuspid repair for FTR (treated [T] group), and 131 (44\%) did not (untreated [UT] group). The preoperative characteristics of the patients are listed in Table 1 . The etiology of MV disease was mainly functional in the UT group $57.7 \%$ vs $33.0 \%, P<.001)$ and the incidence of atrial fibrillation was greater in the T group ( $46.1 \%$ vs $13.7 \%, P<.001)$. The echocardiographic values, as expected, were different between the 2 groups (Table 1).

During follow-up, those patients who showed any sign of repair failure, prosthetic malfunction, or severe impairment of left ventricular function, events that could justify the development of additional FTR, were excluded from the present study $(\mathrm{n}=15)$.

\section{Echocardiographic Evaluation}

All the patients underwent transthoracic echocardiography preoperatively. The tricuspid valve (TV) was evaluated, and the following data were recorded: tricuspid annulus (TA) diameter (apical 4-chamber view) in systole and diastole, the tenting area, and the TV coaptation depth. The severity of FTR, as assessed by Doppler echocardiography, was graded on a scale from 1 to 4 (1, mild; 2 , moderate; 3 , moderate to severe; and 4 , 


$$
\begin{aligned}
& \text { Abbreviations and Acronyms } \\
& \begin{aligned}
\mathrm{CI} & =\text { confidence interval } \\
\mathrm{FTR} & =\text { functional tricuspid regurgitation } \\
\mathrm{HR} & =\text { hazard ratio } \\
\mathrm{MV} & =\text { mitral valve } \\
\text { sysTA } & =\text { systolic dimensions of the tricuspid } \\
& \text { annulus } \\
\mathrm{T} & =\text { treated } \\
\mathrm{TA} & =\text { tricuspid annulus } \\
\mathrm{TR} & =\text { tricuspid regurgitation } \\
\mathrm{TV} & =\text { tricuspid valve } \\
\mathrm{UT} & =\text { untreated }
\end{aligned}
\end{aligned}
$$

severe) ${ }^{3,4}$ The right ventricular dimension in diastole was recorded, as was the tricuspid annular peak systolic excursion. The right ventricular systolic pressures were estimated using continuous-wave Doppler data and the simplified Bernoulli equation $(4 \times$ [peak tricuspid regurgitation [TR] velocity $]^{2}$ ), with $10 \mathrm{~mm} \mathrm{Hg}$ added for the estimated right atrial pressure. The value obtained was considered the systolic pulmonary artery pressure. Also, 20 volunteers were evaluated to have normal echocardiographic values as a reference (Table 2).

\section{Surgical Indications}

Patients with an FTR grade of moderate or greater were always treated. In addition, 16 patients underwent FTR treatment by surgeon choice (because of undergrading during perioperative transesophageal echocardiography). Mild FTR was always treated in the T group when the sysTA was greater than $24 \mathrm{~mm}$ (median value of normal). Patients with a sysTA of $24 \mathrm{~mm}$ or less were not treated, and all had had mild FTR. Thus, the UT group included 131 patients, 115 with mild FTR (81 with sysTA > $24 \mathrm{~mm}$ but not treated because not all the surgeons agreed to treat mild FTR, and 34 with sysTA of $\leq 24 \mathrm{~mm}$ ) and 16 patients with moderate or greater FTR. The T group included 59 patients with mild FTR and sysTA greater than $24 \mathrm{~mm}$ and 108 patients with moderate or greater FTR.

\section{Surgical Details}

All patients underwent surgery with cardiopulmonary bypass and intermittent antegrade/retrograde warm blood cardioplegia. A median sternotomy was used in every case.

The mitral valve was replaced in 100 patients (39 in the UT group and 61 in the T group, $P=.222$ ) and was repaired in the remaining patients. Ablation of atrial fibrillation was performed in 47 patients ( 4 in the UT group and 43 in the T group, $P=.021$ ).

De Vega annuloplasty was performed, starting twice from the anteroseptal commissure and ending twice at the level of the septal annulus in front of the coronary sinus, using 2-0 Ethibond and not monofilament suture. We extended the correction beyond the posteroseptal commissure because the septal leaflet insertion near this commissure is a part of the free wall, which can dilate. ${ }^{5}$ The final orifice was gauged to a No. 25 cylindrical prosthetic sizer. The technique used for band annuloplasty has been previously described. ${ }^{6}$ In brief, the first suture was positioned starting from the anteroseptal commissure and the last one at the septal annulus, $28.5 \mathrm{~mm}$ distant from the first suture and roughly in front of the coronary sinus. The band was $50 \mathrm{~mm}$ long (SMB50, Sorin, Saluggia, Italy), and the final circumference was $78.5 \mathrm{~mm}$ (the circumference of a No. 25 sizer).

From March 2006 to June 2008, all patients underwent doublepledgeted De Vega suture annuloplasty. Since June 2008, some patients with an enlarged TA underwent band annuloplasty $(n=25)$. Over time, the indications became better specified; the current indications are listed in Table 3. De Vega suture annuloplasty was reserved for patients with sysTA, in systole, up to $28 \mathrm{~mm}$ (the maximal systolic value of the sysTA in normal patients), and band annuloplasty was used when the sysTA was larger than $28 \mathrm{~mm}$. Ultimately, the indications for which technique of annuloplasty to use depend on the sysTA dimensions and not on the FTR grade.

\section{Follow-up}

All the surviving patients were followed up at our outpatient clinic and underwent an echocardiographic examination after a mean of $13 \pm 8$ months. The follow-up period ended in March 2009 and was 100\% complete. The median follow-up time was 22 months (interquartile range, 13-37 months), without any difference between the 2 groups.

\section{Statistical Analysis}

The results are expressed as the mean \pm standard deviation in the case of normally distributed continuous variables. In the case of categorical variables, the counts and percentages are reported. Statistical analysis comparing the 2 groups was performed using the unpaired 2-tailed $t$ test for continuous normally distributed variables, with the Mann-Whitney $U$ test for continuous non-normally distributed variables and Pearson's chi-square test (or Fisher's exact test) for categorical variables. The preand postoperative data within each group were compared using a paired $t$ test. The 2-year actuarial outcome was evaluated using the Kaplan-Meier method. Cox analysis was performed to identify the risk factors for FTR recurrence, considered as moderate or greater FTR at the follow-up examination. The results are reported as the hazard ratio (HR), $95 \%$ confidence interval (CI), and $P$ value. For all tests, $P<.05$ was considered significant. The Statistical Package for Social Sciences software (SPSS, Chicago, Ill) was used.

\section{RESULTS}

The 30-day mortality rate was $6.0 \%$ (18 patients) overall, $8.4 \%$ (11 patients) in the UT group, and $4.2 \%$ (7 patients) in the $\mathrm{T}$ group $(P=\mathrm{NS})$. The 2 -year survival rate was $87.0 \% \pm 2.7 \%$ and $83.6 \% \pm 3.3 \%(P=\mathrm{NS})$, and the survival rate stratified by New York Heart Association class I-II was $83.6 \% \pm 3.1 \%$ and $79.2 \% \pm 3.7 \%(P=\mathrm{NS})$ in the $\mathrm{T}$ and UT groups, respectively.

The 256 survivors included in the present study underwent an echocardiographic examination at a mean followup of $13 \pm 8$ months, $14 \pm 8$ months for the T group and $12 \pm 7$ months for the UT group $(P=.038)$. The echocardiographic modifications are listed in Table 4.

The preoperative mean FTR grade was $1.11 \pm 0.32$ in the UT group and $2.11 \pm 0.92$ in the T group $(P<.001)$. At discharge from the hospital, the mean FTR grade had not decreased significantly in the UT group $(0.87 \pm 0.49$, $P=\mathrm{NS}$ ) but had significantly decreased in the T group $(0.45 \pm 0.36, P<.001)$. At follow-up, the mean FTR grade had returned to the preoperative values in the UT group $(1.03 \pm 0.57)$ but had remained similar to the early postoperative values in the $\mathrm{T}$ group $(0.48 \pm 0.32)$.

Only 18 patients $(16.4 \%)$ in the UT group had FTR grade 0 compared with $83(56.8 \%)$ in the T group $(P<.001)$. A total of 24 patients had FTR grade 2 or greater, 16 $(14.5 \%)$ in the UT group and $8(5.5 \%)$ in the $\mathrm{T}$ group 
TABLE 1. Preoperative characteristics

\begin{tabular}{|c|c|c|c|}
\hline & $\begin{array}{l}\text { UT group } \\
(\mathbf{n}=131)\end{array}$ & $\begin{array}{c}\text { T group } \\
(\mathrm{n}=167)\end{array}$ & $\begin{array}{c}P \\
\text { value }\end{array}$ \\
\hline Age $(y)$ & $66 \pm 11$ & $63 \pm 12$ & NS \\
\hline Female gender & $54(41.5)$ & $74(44.3)$ & NS \\
\hline Functional MR & $73(55.7)$ & $55(33.0)$ & $<.001$ \\
\hline Ischemic MR & $59(45.0)$ & $32(19.2)$ & $<.001$ \\
\hline Nonischemic MR & $14(10.7)$ & $23(13.8)$ & NS \\
\hline Structural MR & $37(28.2)$ & $70(41.9)$ & $<.001$ \\
\hline MV stenosis & $17(13.0)$ & $42(25.1)$ & .005 \\
\hline MV prosthesis dysfunction & $4(3.1)$ & 0 & .03 \\
\hline Mean NYHA class & $2.5 \pm 0.7$ & $2.4 \pm 0.7$ & NS \\
\hline Atrial fibrillation & 18 (13.7) & $77(46.1)$ & $<.001$ \\
\hline Logistic EuroSCORE & $8.9 \%$ & $7.9 \%$ & NS \\
\hline TR $1+$ & $115(87.8)$ & $59(35.3)$ & $<.001$ \\
\hline TR $2+$ & $12(9.2)$ & $52(31.1)$ & $<.001$ \\
\hline TR $3+$ & $3(2.3)$ & $45(26.9)$ & $<.001$ \\
\hline TR $4+$ & $1(0.8)$ & $11(6.6)$ & $<.001$ \\
\hline TV annulus in diastole (mm) & $32 \pm 4$ & $37 \pm 5$ & $<.001$ \\
\hline TV annulus in systole (mm) & $25 \pm 4$ & $31 \pm 4$ & .00 \\
\hline Tenting area $\left(\mathrm{cm}^{2}\right)$ & $1.15 \pm 0.35$ & $1.36 \pm 0.27$ & $<.00$ \\
\hline TVCD (mm) & $2.89 \pm 0.65$ & $3.45 \pm 0.47$ & $<.001$ \\
\hline RVD (mm) & $28 \pm 3$ & $31 \pm 5$ & .00 \\
\hline TAPSE (mm) & $19 \pm 4$ & $17 \pm 4$ & .03 \\
\hline SPAP (mm Hg) & $35 \pm 11$ & $44 \pm 14$ & .01 \\
\hline $\mathrm{EF}(\%)$ & $48 \pm 11$ & $47 \pm 13$ & NS \\
\hline $\mathrm{EDD}(\mathrm{mm})$ & $54 \pm 13$ & $54 \pm 10$ & NS \\
\hline $\mathrm{ESD}(\mathrm{mm})$ & $37 \pm 11$ & $38 \pm 9$ & NS \\
\hline
\end{tabular}

$U T$, Untreated; $T$, treated; $N S$, not significant; $M R$, mitral regurgitation; $M V$, mitral valve; $N Y H A$, New York Heart Association; $T R$, tricuspid regurgitation; $T V$, tricuspid valve; $C D$, coaptation depth; $R V D$, right ventricular diameter; TAPSE, tricuspid annular plane systolic excursion; $S P A P$, systolic pulmonary artery pressure; $E F$, ejection fraction; $E D D$, end-diastolic diameter; $E S D$, end-systolic diameter. Data presented as mean $\pm \mathrm{SD}$ or $\mathrm{n}(\%)$.

$(P=.026)$; no patient in the $\mathrm{T}$ group had FTR grade $3+$ to $4+$ versus 3 patients in the UT group. Globally, the FTR grade increased in 12 patients $(10.9 \%)$ in the UT group compared with none in the T group $(P<.001)$.

Of the 110 patients in the UT group who had undergone a postoperative echocardiographic examination, $101 \mathrm{had}$ had mild FTR preoperatively. Of the 101 patients, 31 had had sysTA of $24 \mathrm{~mm}$ or less, and none showed a FTR grade

TABLE 2. Normal echocardiographic values in 20 volunteers

\begin{tabular}{lccccc}
\hline \multicolumn{1}{c}{ Variable } & Mean & SD & Median & Minimum & Maximum \\
\hline $\mathrm{BS}\left(\mathrm{m}^{2}\right)$ & 1.81 & 0.16 & 1.85 & 1.49 & 2.16 \\
$\mathrm{BMI}\left(\mathrm{kg} / \mathrm{m}^{2}\right)$ & 24.3 & 2.2 & 24.3 & 21.0 & 29.0 \\
Age $(\mathrm{y})$ & 41.3 & 8.8 & 44 & 27 & 53 \\
RV (diastole) $(\mathrm{mm})$ & 27.6 & 1.5 & 28 & 24 & 30 \\
TA (systole) $(\mathrm{mm})$ & 24.5 & 2.0 & 24 & 21 & 28 \\
TA (diastole) (mm) & 27.4 & 2.0 & 27 & 24 & 31 \\
Systolic-diastolic & 10.5 & 3.7 & 10.3 & 5.6 & 20.7 \\
$\quad$ modification $(\%)$ & & & & & \\
TVCD (mm) & 2.76 & 0.59 & 2.70 & 1.80 & 4.20 \\
Tenting area $\left(\mathrm{cm}^{2}\right)$ & 0.93 & 0.20 & 0.88 & 0.69 & 1.36 \\
\hline
\end{tabular}

$S D$, standard deviation; $B S$, body surface; $B M I$, body mass index; $R V$, right ventricle; $T A$, tricuspid annulus; $T V C D$, tricuspid valve coaptation depth.
TABLE 3. Echocardiographic-based indications for treatment of functional tricuspid regurgitation

\begin{tabular}{|c|c|c|c|}
\hline \multirow[b]{2}{*}{ FTR } & \multicolumn{3}{|c|}{ Systolic TA grade (apical 4-chamber view) } \\
\hline & $\leq \mathbf{2 4} \mathbf{~ m m}$ & 25-28 mm & $>28 \mathrm{~mm}$ \\
\hline Mild & No surgery & De Vega & Band \\
\hline Moderate or greater & De Vega?* & De Vega & Band \\
\hline
\end{tabular}

FTR, functional tricuspid regurgitation; $T A$, tricuspid annulus. *No patient had FTR of moderate or greater and TA grade of $\leq 24 \mathrm{~mm}$.

increase. Of the 70 with a sysTA greater than $24 \mathrm{~mm}, 7$ showed a FTR grade increase to moderate or greater. Of the 9 patients with a preoperative FTR grade of moderate or greater, all had had sysTA greater than $24 \mathrm{~mm}$ and all maintained the FTR grade. Cox regression analysis showed that, in the UT group, a sysTA greater than $24 \mathrm{~mm}$ was a risk factor for a greater FTR grade during follow-up (HR, 2.4; $95 \%$ CI, 1.4-5.1; $P=.020$ ).

Of the 256 survivors, 24 had a postoperative FTR of grade 2 or more, which was considered an event. Proportional Cox regression analysis evidenced only 2 risk factors: inclusion in the UT group (HR, $4.1 ; 95 \% \mathrm{CI}, 1.7-9.7 ; P=.002)$ and atrial fibrillation persistence at 6 months (HR, $2.0 ; 95 \% \mathrm{CI}$, $1.1-4.6 ; P=.029)$. Nevertheless, patients with atrial fibrillation persistence had a greater FTR grade in the UT group $(\mathrm{n}=10)$ than in the $\mathrm{T}$ group $(\mathrm{n}=33 ; 1.6 \pm 0.7$ vs $0.91 \pm$ 0.63 , respectively, $P=.005$ ), showing the protective effect of tricuspid annuloplasty in this subgroup of patients. The incidence of preoperative atrial fibrillation was greater in the $\mathrm{T}$ group, but it was not a risk factor.

The sysTA was greater than $28 \mathrm{~mm}$ in all patients in the $\mathrm{T}$ group $(\mathrm{n}=8)$ with a follow-up FTR grade of 2 or more. All 8 patients had undergone De Vega annuloplasty. This finding supports the concept that a careful analysis of the echocardiographic anatomy could be crucial for choosing the correct strategy (band vs De Vega annuloplasty) and that De Vega annuloplasty can have suboptimal results when the sysTA is greater than $28 \mathrm{~mm}$, but not when it is $28 \mathrm{~mm}$ or less.

In the 16 patients in the UT group who had, at follow-up, a FTR grade of 2 or more, most (10/16) had a sysTA of 25 to $28 \mathrm{~mm}$, supporting the strategy to correct any FTR grade if the sysTA is $24 \mathrm{~mm}$ or greater. The remaining 6 patients had a sysTA greater than $28 \mathrm{~mm}$. Preoperatively, 7 of these patients had had FTR grade 1 and 9 had had FTR grade 2 or more.

\section{DISCUSSION}

The main findings of the present study were that when FTR is not treated, the FTR grade will have increased in $10.9 \%$ of patients after 1 year. Also, extensive treatment of FTR of any grade resulted in an early reduction of the FTR grade that was sustained after 1 year. Third, the echocardiographic TA systolic dimension will be crucial for decision making, because the TA dimensions correlated with 
TABLE 4. Echocardiographic modifications in 256 survivors

\begin{tabular}{|c|c|c|c|c|c|c|}
\hline \multirow[b]{2}{*}{ Variable } & \multicolumn{3}{|c|}{ UT group $(n=110)$} & \multicolumn{3}{|c|}{$T$ group $(n=146)$} \\
\hline & Preoperative & Postoperative & $P$ value & Preoperative & Postoperative & $P$ value \\
\hline $\mathrm{TV}$ annulus in diastole $(\mathrm{mm})$ & $31 \pm 3$ & $29 \pm 3$ & NS & $37 \pm 4$ & $26 \pm 3$ & $<.001$ \\
\hline TV annulus in systole (mm) & $25 \pm 4$ & $24 \pm 3$ & NS & $31 \pm 4$ & $22 \pm 3$ & $<.001$ \\
\hline Tenting area $\left(\mathrm{cm}^{2}\right)$ & $1.0 \pm 0.6$ & $0.8 \pm 0.6$ & NS & $1.4 \pm 0.7$ & $0.7 \pm 0.3$ & $<.001$ \\
\hline TVCD (mm) & $7.2 \pm 5.4$ & $7.0 \pm 5.5$ & NS & $8.3 \pm 3.9$ & $6.3 \pm 3.3$ & $<.001$ \\
\hline RVD (mm) & $28 \pm 4$ & $28 \pm 6$ & NS & $30 \pm 4$ & $28 \pm 4$ & NS \\
\hline TAPSE (mm) & $19 \pm 4$ & $15 \pm 3$ & .001 & $18 \pm 4$ & $16 \pm 3$ & NS \\
\hline SPAP (mm Hg) & $34 \pm 9$ & $30 \pm 8$ & NS & $44 \pm 14$ & $32 \pm 12$ & .001 \\
\hline $\mathrm{EF}(\%)$ & $48 \pm 7$ & $47 \pm 9$ & NS & $44 \pm 12$ & $48 \pm 11$ & NS \\
\hline MR (from 1 to 4 ) & $2.7 \pm 1.1$ & $0.4 \pm 0.5$ & $<.001$ & $2.9 \pm 1.1$ & $0.7 \pm 0.9$ & $<.001$ \\
\hline TR (from 0 to 4 ) & $1.1 \pm 0.4$ & $1.0 \pm 0.6$ & NS & $2.0 \pm 0.9$ & $0.5 \pm 0.6$ & $<.001$ \\
\hline No TR & 0 & $18(16.4)$ & $<.001$ & 0 & $83(56.8)$ & $<.001$ \\
\hline TR $1+$ & $101(91.8)$ & $76(69.1)$ & $<.001$ & $54(37.0)$ & 55 (37.7) & NS \\
\hline TR $2+$ & 7 (6.4) & $13(11.8)$ & NS & $45(30.8)$ & $8(5.5)$ & $<.001$ \\
\hline TR 3+14+ & $2(1.8)$ & $3(2.7)$ & NS & $47(32.2)$ & 0 & $<.001$ \\
\hline Follow-up (mo) & $12 \pm 7$ & & & & $14 \pm 8$ & .038 \\
\hline
\end{tabular}

Abbreviations as in Table 1. Data presented as mean \pm SD or $\mathrm{n}(\%)$.

the regurgitant volume ${ }^{7}$ and annular dilation, but not with pulmonary hypertension, the right ventricular dimension, or tricuspid tenting. Thus, the TA dimensions were the best FTR determinant. ${ }^{8}$ Finally, annuloplasty was able to reduce the amount of FTR in patients with postoperative atrial fibrillation.

TR is a common echocardiographic finding. Nath and colleagues $^{1}$ evaluated 5223 patients who had undergone echocardiography at 1 laboratory within a 4-year period. The TR incidence was $88.5 \%$, with $15.5 \%$ having moderate or greater TR. These patients, after adjustment for left ventricular ejection fraction, inferior vena cava size, and right ventricular size and function, had a 4-year survival worse than that of patients without TR.

The evolution of untreated FTR was studied by different investigators. Dreyfus and colleagues ${ }^{2}$ corrected the FTR in 311 patients according to the anatomic criteria (intraoperative diameter $\geq 70 \mathrm{~mm}$ ). Before surgery, of the 148 patients in whom tricuspid annuloplasty was performed, 38 (25.7\%) had no FTR and $92(62.2 \%)$ had grade 1 FTR. After a mean follow-up of 4.8 years, the FTR grade had changed from 0.88 to 0.36 in the treated patients and from 0.82 to 2.07 in the untreated patients $(P<.001)$, with $48 \%$ of the patients having an increase to FTR grade 2 or more. These findings had no influence on survival or cardiac event-free survival. Matsuyama and colleagues ${ }^{9}$ followed up 174 patients without FTR repair for a mean of 8.2 years. Of the 174 patients, $24 \%$ had had FTR grade 2 and $76 \%$ had had grade 1 or less. During follow-up, $16 \%$ had developed FTR of grade 3 or more. Preoperative FTR grade 2 was a risk factor for the late development of FTR grade 3 or more (odds ratio, $3.9 ; P=.004$ ).

Although untreated FTR can worsen over time and can be associated with reduced survival, the results of surgical correction of FTR have not been perfect.
McCarthy and colleagues ${ }^{10}$ evaluated 790 patients who had undergone FTR treatment using different techniques (rigid complete ring, incomplete band, De Vega, and pericardium) and had echocardiographic reports after a median of 6 months. They found that residual FTR occurred in 14\% of patients in the first month after operation for all types of annuloplasty. The FTR grade evolved differently according to the repair technique. The severity of regurgitation remained stable over time after repair with the complete rigid ring and with a band, but it increased significantly with the De Vega and pericardium repair techniques.

Kuwaki and colleagues ${ }^{11}$ found that $25 \%$ of the 194 patients treated with De Vega annuloplasty had had, in the first postoperative months, FTR recurrence of a moderate or greater grade. The risk factor was preoperative FTR grade 4. Fukuda and colleagues ${ }^{12}$ followed up with sequential echocardiograms in 39 patients, who had undergone different annuloplasty techniques. Of the 39 patients, $23 \%$ developed moderate or greater FTR recurrence in the first postoperative month and $31 \%$ after 20 months. The risk factors for late FTR return were reduced postoperative left ventricular function (cutoff value, $36.6 \%$ ), early postoperative FTR (cutoff value for maximal TR area/right atrial area ratio greater than 0.13 ). These investigators introduced an interesting concept, because they found preoperative tenting height (cutoff value, $0.51 \mathrm{~cm}$ ) and preoperative tenting area (cutoff value, $0.80 \mathrm{~cm}^{2}$ ) to be risk factors for FTR. They emphasized that the cause of FTR return could be a ventricular factor and not technical failure. Although the TV coaptation depth was coherent with our measures (Tables 1 to 3 ), the tenting area was smaller than we measured, very likely because of technical issues.

De Vega annuloplasty has been found in different reports to be a technical risk factor for FTR return. ${ }^{13,14}$ Our personal experience ${ }^{15}$ did not confirm these results. Two 
groups of 50 propensity matched patients, with a FTR grade of moderate or more, underwent MV surgery for functional mitral regurgitation. In the first group, the FTR was treated (De Vega annuloplasty) and in the second was untreated. At 1 year after surgery, the FTR grade had decreased in the treated group from $3.1 \pm 0.7$ to $0.7 \pm 0.7(P<.001)$ and had remained stable in the untreated group (from $2.2 \pm 0.4$ to $2.3 \pm 0.9, P=\mathrm{NS}$ ). Thus, De Vega annuloplasty provided good midterm results.

The published data have suggested that moderate or greater FTR is a risk factor for survival and that any grade of FTR can worsen over time, independently of the initial grade. However, the results of De Vega suture annuloplasty have been debatable, with most studies showing a greater incidence of FTR recurrence compared with device-based annuloplasty. Finally, early recurrence of FTR seems to be frequent, irrespective of the technique used.

These findings pushed us to a more aggressive approach for FTR correction, determined mainly from the preoperative TA echocardiographic systolic dimensions. Regarding functional mitral regurgitation, we believe that the systolic, rather than the diastolic, dimensions of the TA are helpful in decision making, because FTR occurs during systole. We decided to also correct mild FTR, because it was evident from the results from Dreyfus and colleagues, ${ }^{2}$ that, even in a population of patients with low-grade or no FTR, the possibility of the FTR worsening over time is high. The correlation between residual FTR and the persistence of atrial fibrillation is well known ${ }^{16}$; nevertheless, tricuspid annuloplasty is able to reduce its progression.

We believe that TA dilation, by itself, cannot be the only determinant of the surgical indication. In fact, $65 \%$ of patients without TA dilation (mean systolic TA dimension $25.4 \pm 3.2$ ) can have FTR grade 1 or $2^{17}$ and are a part of a subgroup whose natural history is worse than that of patients without FTR. Chopra and colleagues ${ }^{18}$ found that in patients with nonsevere FTR (jet area/right atrium area ratio $<33 \%$; mean, $27.5 \pm 6.9$ ), the mean TA diastolic dimension was $17.8 \pm 2.5 \mathrm{~mm}$. This value was equivalent to roughly $32 \mathrm{~mm}$ in a normal patient, $1 \mathrm{~mm}$ more than the maximal value we found in our normal population (Table 2). Thus, many patients can have significant FTR even if the TA dimensions are within the normal range.

In agreement with these published data, we found that most of the patients in the UT group who, during followup, had an FTR grade of moderate or greater still had a sysTA within the normal range $(25-28 \mathrm{~mm})$. This finding justifies our strategy to correct FTR when the sysTA is in the higher normal range $(>24 \mathrm{~mm})$. In contrast, the patients with sysTA of $24 \mathrm{~mm}$ or less did not show any increase in FTR grade, at least in the midterm.

De Vega annuloplasty (not limited to the posteroseptal commissure but extended to the insertion of the septal leaflet in front of the coronary sinus) can reasonably be performed in patients with a less dilated sysTA $(\leq 28 \mathrm{~mm})$; however, in patients with a more dilated sysTA ( $>29 \mathrm{~mm}$ ), it is very likely that band annuloplasty would be the best option. This speculation was supported by the retrospective analysis of patients in the $\mathrm{T}$ group with a postoperative FTR grade of 2 or greater. All these had undergone De Vega annuloplasty and had a sysTA greater than $29 \mathrm{~mm}$. However, no patients who had undergone De Vega annuloplasty and sysTA of $28 \mathrm{~mm}$ or less showed FTR grade progression. This solution can be cost effective, because it allows the expansion of the indications for TV annuloplasty without the necessity of using a band in every case.

The TV coaptation depth evaluation, a new concept introduced by Fukuda and colleagues, ${ }^{12}$ can be useful in the decision making of tricuspid surgery. Very likely, techniques different from those commonly used are needed, ${ }^{19,20}$ even if the problem is not yet well defined.

\section{Study Limitations}

The present study was retrospective, the subgroup population was small, and the follow-up was relatively short. These were its main limitations. Moreover, the differences in the postoperative FTR grade were small and potentially clinically irrelevant. The 2 groups were also different; however, the New York Heart Association class, ejection fraction, and left ventricular dimensions were similar, and in every case, the cause of FTR was functional. Moreover, more patients in the UT group had functional mitral regurgitation, but the systolic pulmonary artery pressure and right ventricular dimensions were lower than those in the $\mathrm{T}$ group. In contrast, group $\mathrm{T}$ had a greater incidence of preoperative atrial fibrillation. However, Cox analysis did not identify either the etiology of mitral disease or preoperative atrial fibrillation as a risk factor. Nevertheless, we cannot foresee the change in FTR grade in the long term when stratified by the etiology of the MV disease, even if progression of FTR, if untreated, was reported after surgery for both mainly structural ${ }^{2}$ and functional ${ }^{15} \mathrm{MV}$ disease. Another important point is that the concept that surgical treatment of a low FTR grade could improve, in the long term, the clinical results is only speculative and was not supported by the data from our short follow-up.

\section{CONCLUSIONS}

We believe that aggressive correction of FTR can be helpful in reducing, in the midterm, the FTR grade, because in patients with preoperative FTR, even of low grade, mitral surgery alone was not able to eliminate or reduce the FTR grade. In the UT group, $10.9 \%$ of patients had a worsened FTR grade and only $12.7 \%$ of them had no FTR. In contrast, in the T group, no patient had a worsened FTR grade and $61.0 \%$ had no FTR, even if the preoperative FTR grade had been greater than in the UT group. We believe that preoperative echocardiographic evaluation of the TA systolic 
dimensions will be helpful to both select patients who need FTR treatment and the proper surgical strategy (Table 3). However, at least in the midterm, the clinical outcomes were not influenced by the surgical strategy.

The natural history of untreated FTR is one of progressive worsening over time, even if the preoperative FTR grade was mild. ${ }^{2}$ Limiting this unfavorable outcome, because the surgical procedure is rapid and effective, must be our goal during MV surgery.

\section{References}

1. Nath J, Foster E, Heidenreich PA. Impact of tricuspid regurgitation on long-term survival. J Am Coll Cardiol. 2004;43:405-9.

2. Dreyfus GD, Corbi PJ, Chan KM, Bahrami T. Secondary tricuspid regurgitation or dilatation: which should be the criteria for surgical repair? Ann Thorac Surg. 2005;79:127-32.

3. González Vílchez F, Zarauza J, Vázquez de Prada JA, Martin Durán R, Ruano J, Delgado C, et al. Assessment of tricuspid regurgitation by Doppler color flow imaging: angiographic correlation. Int J Cardiol. 1994;44: 275-83.

4. Zoghbi WA, Enriquez-Sarano M, Foster E, Grayburn PA, Kraft CD, Levine RA, et al. American Society of Echocardiography. Recommendations for evaluation of the severity of native valvular regurgitation with two-dimensional and Doppler echocardiography. J Am Soc Echocardiogr. 2003;16:777-802.

5. Ton-Nu T-T, Levine RA, Handshumaker MD, Dorer DJ, Yosefy C, Fan D, et al. Geometric determinants of functional tricuspid regurgitation: insights from 3-dimensional echocardiography. Circulation. 2006;114:143-9.

6. Calafiore AM, Iacò AL, Contini M, Di Mauro M. A single-size band, $50 \mathrm{~mm}$ long, for tricuspid annuloplasty. Eur J Cardiothorac Surg. 2008;34:677-9.

7. Sugimoto T, Okada M, Ozaki N, Hatakeyama T, Kawahira T. Long-term evaluation of treatment for functional tricuspid regurgitation with regurgitant volume: characteristic differences based on primary cardiac lesion. J Thorac Cardiovasc Surg. 1999;117:463-71.

8. Sagie A, Schwammenthal E, Padial LR, Vazquez de Prada JA, Weyman AE, Levine RA. Determinants of functional tricuspid regurgitation in incomplete tricuspid valve closure: Doppler color flow study of 109 patients. J Am Coll Cardiol. 1994;24:446-53.

9. Matsuyama K, Matsumoto M, Sugita T, Nishizawa J, Tokuda Y, Matsuo T. Predictors of residual tricuspid regurgitation after mitral valve surgery. Ann Thorac Surg. 2003;75:1826-8.

10. McCarthy PM, Bhudia SK, Rajeswaran J, Hoercher KJ, Lytle BW, Cosgrove DM, et al. Tricuspid valve repair: durability and risk factors for failure. J Thorac Cardiovasc Surg. 2004;127:674-85.

11. Kuwaki K, Morishita K, Tsukamoto M, Abe T. Tricuspid valve surgery for functional tricuspid valve regurgitation associated with left-sided valvular disease. Eur J Cardiothorac Surg. 2001;20:577-82.

12. Fukuda S, Gillinov AM, McCarthy PM, Stewart WJ, Song JM, Kihara T, et al Determinants of recurrent or residual functional tricuspid regurgitation after tricuspid annuloplasty. Circulation. 2006;114:I582-7.

13. Tang GH, David TE, Singh SK, Maganti MD, Armstrong S, Borger MA. Tricuspid valve repair with an annuloplasty ring results in improved long-term outcomes. Circulation. 2006;114:I577-81.

14. Matsuyama K, Matsumoto M, Sugita T, Nishizawa J, Tokuda Y, Matsuo T, et al. De Vega annuloplasty and Carpentier-Edwards ring annuloplasty for secondary tricuspid regurgitation. J Heart Valve Dis. 2001;10:520-4.

15. Calafiore AM, Gallina S, Iacò AL, Contini M, Bivona A, Gagliardi M, et al Mitral valve surgery for functional mitral regurgitation: should moderateor-more tricuspid regurgitation be treated? A propensity score analysis. Ann Thorac Surg. 2009;87:698-703.

16. Kim H-K, Kim Y-J, Kim K-I, Jo S-H, Kim K-B, Ahn H, et al. Impact of the maze operation combined with left-sided surgery on the change in tricuspid regurgitation over time. Circulation. 2005;112(suppl I):I-14-9.

17. Anwar MA, Geleijnse ML, ten Cate FJ, Meijboom FJ. Assessment of tricuspid valve annulus size, shape and function using real-time three-dimensional echocardiography. Interact CardioVasc Thorac Surg. 2006;5:683-7.

18. Chopra HK, Nanda NC, Fan P, Kapur KK, Goyal R, Daruwalla D, et al. Can twodimensional echocardiography and Doppler flow mapping identify the need for tricuspid repair? J Am Coll Cardiol. 1989;14:1266-74.

19. De Bonis M, Lapenna E, La Canna G, Grimaldi A, Maisano F, Torracca L, et al. A novel technique for correction of severe tricuspid valve regurgitation due to complex lesions. Eur J CardioThoracic Surg. 2004;25:760-5.

20. Dreyfus GD, Raja SG, Chan KMJ. Tricuspid leaflet augmentation to address severe tethering in functional tricuspid regurgitation. Eur J Cardiothorac Surg. 2008;34:908-10. 